\title{
The Kinematic and Dynamic Study of an Inertial Propulsion System Based on Rotating Masses
}

\section{Attila Geröcs ${ }^{1}$, Zoltan-Iosif Korka ${ }^{1}$, István Bíró ${ }^{2}$ Dorian Nedelcu ${ }^{1}$}

${ }^{1}$ University "Eftimie Murgu" of Resita, Faculty of Engineering and Management, Traian Vuia Sq., no.1-4, 320085 Resita, Romania, e-mails: a.gerocs@uem.ro, z.korka@uem.ro,d.nedelcu@uem.ro

${ }^{2}$ University of Szeged, Faculty of Engineering, Mars tér 7, H-6724 Szeged, Hungary, e-mail: biro-i@mk.u-szeged.hu

Abstract: The present paper is dedicated to the investigation of the kinematic and dynamic behavior of an inertial propulsion system, using rotating masses. The analytical results were compared with the results of a simulation using the SolidWorks Motion software. The following elements were considered: positional analysis, kinematic analysis of velocities and dynamic analysis.

\section{Introduction}

Since the beginning of the Industrial Revolution, researchers and inventors from around the world, have invested energy and creativity into developing innovative propulsion systems, which use the centrifugal force of rotating masses to generate propulsive force and linear motion. Even if we are talking about previous [1] or recent [2-6] pursuits, regrettably, only a few of these machines have been constructed, and even less employed for practical application. These devices are known under different names, such as: inertial propulsion engines, inertial drives, impulse engines, non-linear propulsion systems, reactionless drives etc., but unfortunately, they were not universally accepted by the traditional core of the scientific community.

Classifications of these propulsion systems can be done according to various criteria. A first point of comparison takes into consideration the type of inertial force. Therein, following types of devices may be treated: 
- Devices which use centrifugal force [3], [7] to generate linear motion

- Devices which use the inertial force resulting from the alternative translation motion [8], [9]

- Devices which transform continuous rotation into discontinuous rotation motion [10]

- Devices which use the rotational motion of various simple inertial rotary motion mechanisms [7]

Depending on the source of the propulsion energy:

- Devices using mechanical energy [2], [11]

- Devices using both mechanical and electromechanical energy [12]

- Devices using propulsion energy [13]

Conditioned on the state of the body in which the inertia force appears, the inertial propulsion systems can be classified into:

- Devices where the inertial force is generated by a solid

- Devices where the force of inertia is generated by a liquid [14].

The aim of the present paper is to analyze the kinematic and dynamic behavior of an inertial propulsion system (IPS) developed by the authors [15], [16]. In this respect, starting from the equations of the geometrical coordinates of the balls, their velocities and accelerations were deducted and calculated for a specific case. In parallel, using the facilities of the Motion module from SolidWorks (SW) software, a kinematic and dynamic simulation was performed, the obtained results being compared with the analytical data. As the obtained results are promising, the authors are encouraged to continue their investigations in the direction of optimizing the system.

\section{Geometry and Main Parameters of the Inertial Propulsion System}

The investigated IPS is based on developing a resultant centrifugal force, operating in the movement direction of the system. The construction of the IPS is presented in Figure 1 and consists of two identical constructive groups, placed in mirror relative to the movement direction. Each group is composed from 8 identical balls (1-8) made from "Plan Carbon Steel", having the radius $r=9 \mathrm{~mm}$ and being placed between two rotating disks (9) with a diameter $\varnothing=280 \mathrm{~mm}$, which are foreseen with 8 radial slots. 
The circular path of the balls is ensured by the inner bore of a retaining disk (10), having an outer, respective an inner diameter $\emptyset_{\text {out } / \text { in }}=410 / 185 \mathrm{~mm}$. The center $\mathrm{O}_{2}$ of the disk (10) is displaced eccentrically relative to the center $\mathrm{O}_{1}$ of the disks (9). The eccentricity $\mathrm{e}$ is in the $\mathrm{Y}$ movement direction of the system. The two constructive groups are placed inside a box (11) made from 1.0037 (S235JR) steel, having the main dimensions $890 \times 570 \times 110 \mathrm{~mm}$. The box (11) is placed on a horizontal table (12) made from the same material grade as the box and having the main dimensions 890x600x10 mm.

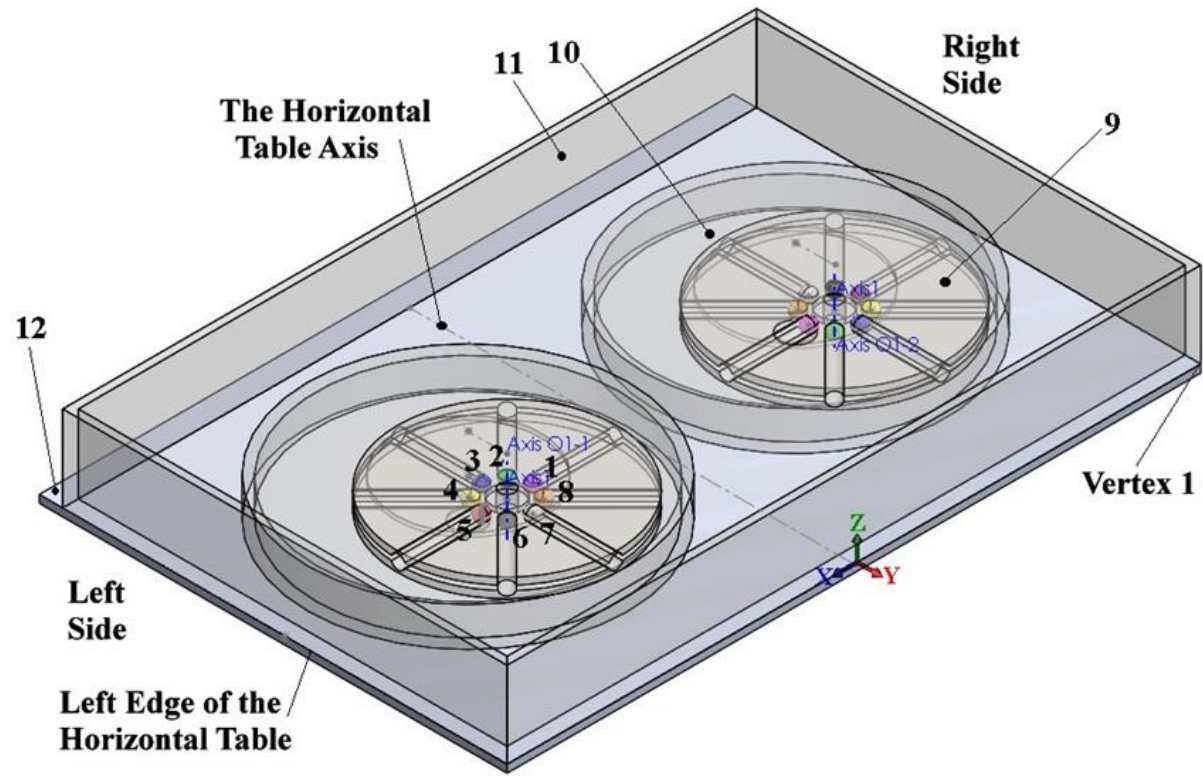

Figure 1

Construction of the IPS

In order to drive the two constructive groups in rotational motion with the same speed, but in opposite directions, a pair of spur gears with transmission ratio 1 was imposed between the two slotted disks (9). The system is driven by a rotary motor applied to the right slotted disk with a constant rotational speed $n_{1}=1500 \mathrm{rot} / \mathrm{min}$, which corresponds to an angular velocity $\omega_{1}=157.08 \mathrm{rad} / \mathrm{s}$, respective 9000 degrees/second.

The operating principle of the IPS bases on the development of a propulsive force as a reaction to the centrifugal forces which are acting on the 8 steel balls. These centrifugal forces may be expressed as:

$$
F_{c_{i}}=m_{0} \cdot \omega_{I}^{2} \cdot R(t)
$$

where $m_{o}$ is the mass of the balls, $\omega_{I}$ the angular velocity of the slotted disks and $R(t)$ the trajectory radius of ball $i$. 


\section{Analytical Investigation of the IPS}

For calculating the physical quantities that characterize the kinematics of the system, one of the balls with the center in $\mathrm{C}_{\mathrm{i}}$ was considered. Further, to the slotted disk (9) a Cartesian system denoted with $\mathrm{xO}_{1} \mathrm{y}$ was attached, while the center $\mathrm{O}_{2}$ of the retaining disk (10) is placed eccentric at the distance $e$. (see Figure 2).

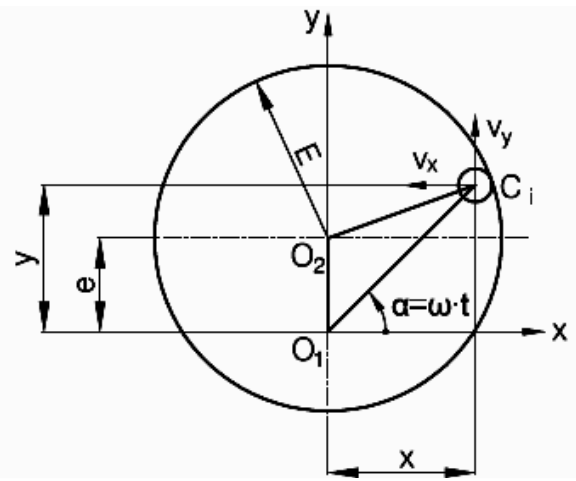

Figure 2

Kinematic of the steel balls

As, during the rotation of the slotted disk, the steel balls (i=1-8) are in contact to the inner bore of the retaining disk (tangent to the circle with radius $E$ ), with the notations from Figure 2, the coordinates of the center $\mathrm{C}_{\mathrm{i}}$ may be expressed as:

$x(t)=R(t) \cos \omega t$ and $y(t)=R(t) \sin \omega t$

Applying the generalized theorem of Pythagoras in the triangle $\mathrm{O}_{1} \mathrm{O}_{2} \mathrm{C}_{\mathrm{i}}$, the trajectory radius $R(t)=O_{l} C_{i}$ can be computed as:

$R(t)=e \sin \omega t+\left[(E-r)^{2}-e^{2} \cos ^{2} \omega t\right]^{1 / 2}$

As a consequence, the coordinates of the center $\mathrm{C}_{\mathrm{i}}$ become:

$x(t)=\frac{e}{2} \sin 2 \omega t+\left[(E-r)^{2}-e^{2} \cos ^{2} \omega t\right]^{1 / 2} \cos \omega t$

and: $y(t)=e \sin ^{2} \omega t++\left[(E-r)^{2}-e^{2} \cos ^{2} \omega t\right]^{1 / 2} \sin \omega t$

Deriving the coordinates $x(t)$ and $y(t)$, the components of the ball velocity along the $x$ and $y$ axis can be expressed as:

$$
\begin{aligned}
& v_{x}(t)=\omega e \cos 2 \omega t-\left[(E-r)^{2}-e^{2} \cos ^{2} \omega t\right]^{1 / 2} \omega \sin \omega t+ \\
& +\left[(E-r)^{2}-e^{2} \cos ^{2} \omega t\right]^{-1 / 2} \frac{\omega e^{2} \cos \omega t \cdot \sin 2 \omega t}{2}
\end{aligned}
$$




$$
\begin{aligned}
& v_{y}(t)=\omega e \sin 2 \omega t+\left[(E-r)^{2}-e^{2} \cos ^{2} \omega t\right]^{1 / 2} \omega \cos \omega t+ \\
& +\left[(E-r)^{2}-e^{2} \cos ^{2} \omega t\right]^{-1 / 2} \frac{\omega e^{2} \sin \omega t \cdot \sin 2 \omega t}{2}
\end{aligned}
$$

Additionally, deriving the upper formulations of the velocities, the components of the acceleration are attained as:

$$
\begin{aligned}
a_{x}(t)= & -2 \omega^{2} e \sin 2 \omega t-\left[(E-r)^{2}-e^{2} \cos ^{2} \omega t\right]^{1 / 2} \omega^{2} \cos \omega t+ \\
& +\left[(E-r)^{2}-e^{2} \cos ^{2} \omega t\right]^{-1 / 2} \omega^{2} e^{2} \cos 3 \omega t- \\
- & {\left[(E-r)^{2}-e^{2} \cos ^{2} \omega t\right]^{-3 / 2} \frac{\omega^{2} e^{4} \cos \omega t \cdot \sin ^{2} 2 \omega t}{8} } \\
a_{y}(t)= & 2 \omega^{2} e \cos 2 \omega t-\left[(E-r)^{2}-e^{2} \cos ^{2} \omega t\right]^{1 / 2} \omega^{2} \sin \omega t+ \\
& +\left[(E-r)^{2}-e^{2} \cos ^{2} \omega t\right]^{-1 / 2} \omega^{2} e^{2} \sin \omega t- \\
- & {\left[(E-r)^{2}-e^{2} \cos ^{2} \omega t\right]^{-3 / 2} \frac{\omega^{2} e^{4} \sin \omega t \cdot \sin ^{2} 2 \omega t}{8} }
\end{aligned}
$$

\section{Simulation of the IPS Functioning}

For the present work, the facilities of the Motion module from SolidWorks software were used. Forwards there are presented the details regarding the generation of the parts geometry, the assembly and the stages of the motion study.

\subsection{Creation of the Parts Geometry and Assembly Mechanism}

The 3D assembly of the IPS mechanism is shown in Figure 1 and detailed in a previous paper [17]. In order to reduce the computing time [18], a simplified geometry was used for simulation. Thus, Figure 3 shows the upper and lower slotted disks (9), which were generated as a single part. Here, the Front Plane, the Central Axis, the Point on Central Axis and the Points $1 \div 8$ were used to apply mates in the IPS assembly. The Points $1 \div 8$ were placed in the central points of the radial slots semispheres. 


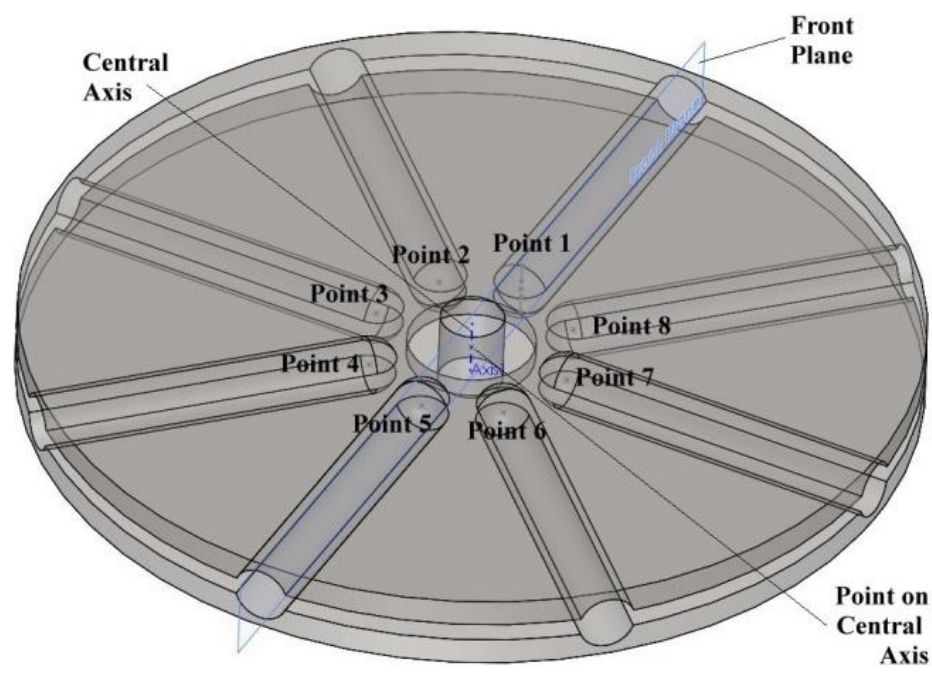

Figure 3

Generation of the slotted disks (9)

Figure 4 shows the retaining disk (10) and the box (11) generated as a single part were the box Axis, the axis $\mathrm{O}_{1-1}, \mathrm{O}_{1-2}$ and point 1 were used to apply mates in the IPS assembly.

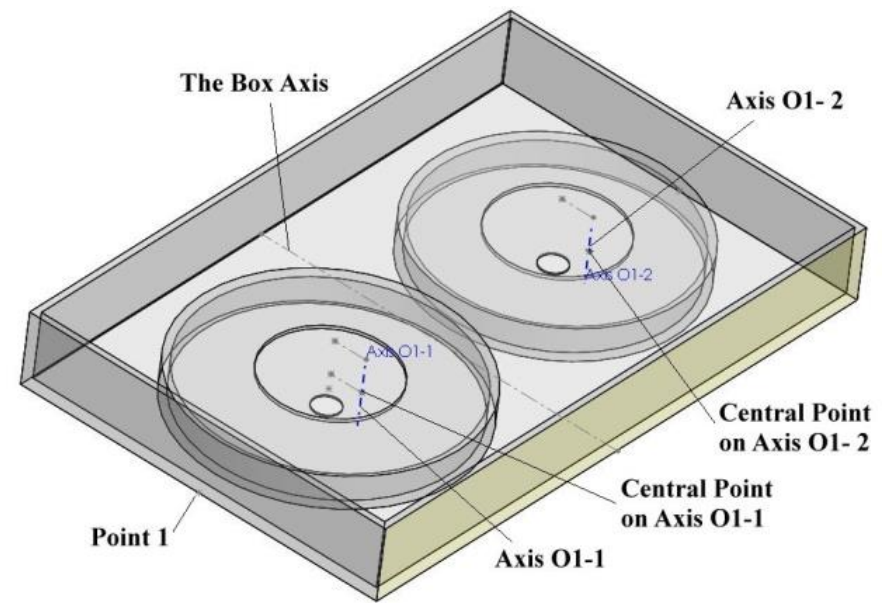

Figure 4

Retaining disk (10) and the box (11) generated as a single part

The components were placed in the assembly with Insert Components command from Assembly toolbar. A part or assembly may be selected from the Part/Assembly to Insert list, while to open an existing part, Browse command must be used. Next, for placing a component, within the graphic area has to be 
click or choosing $\checkmark$ to place the component origin coincident with the assembly origin. Only the horizontal table (12) was placed with the origin coincident with the assembly origin. By default, the first part placed in an assembly is fixed and has a (f) mark placed before its name in the FeatureManager design tree. The other components were placed without this restriction.

\subsection{Stages of the Motion Study}

Following stages [19], [20] were used in the motion study:

- Activation of the SolidWorks Motion module

- Creation and specification of the study's options

- Specification of Rotary Motor

- Specification of Gravity

- Specification of SolidBody Contacts

- Specification of the Mates

- Running the design study

To specify the rotary motor, Motor icon was chosen, while the inner cylindrical face of the slotted disk (9) was selected, together with Constant speed from Motor Type list. As previously stated, a value of $1500 \mathrm{rpm}$ was set in the speed motor field. Choosing $\checkmark$, the Rotary Motor 1 branch was created in the Motion Manager design tree.

Further, selecting Gravity icon, axis $\mathrm{Z}$ as direction of action and the value of $9806.65 \mathrm{~mm} / \mathrm{s}^{2}$, the gravitational forces acting on the mechanism were simulated. No friction between the components was imposed. Finally, the mates indicated in Table 1 were applied in the motion study between the components of the assembly.

Table 1

Mates applied to the IPS mechanism

\begin{tabular}{|c|c|c|c|}
\hline Mate name & Mate type & Component 1 & Component 2 \\
\hline Mate 1 & Coincident & Box axis & Horizontal table axis \\
\hline Mate 2 & Coincident & Point 1 of the box axis & $\begin{array}{l}\text { Left edge of the } \\
\text { horizontal table }\end{array}$ \\
\hline Mate 3 & Coincident & $\begin{array}{l}\text { Axis } \mathrm{O}_{1-1} \text { of the left } \\
\text { retaining disk (10) }\end{array}$ & $\begin{array}{l}\text { Central axis of the left } \\
\text { rotating disk (9) }\end{array}$ \\
\hline Mate 4 & Coincident & $\begin{array}{c}\text { Central Point on axis } \mathrm{O}_{1-1} \text { of } \\
\text { the left retaining disk (10) }\end{array}$ & $\begin{array}{l}\text { Point on central axis of } \\
\text { the left rotating disk (9) }\end{array}$ \\
\hline Mate 5 & Coincident & $\begin{array}{l}\text { Axis } \mathrm{O}_{1-2} \text { of the } \\
\text { right retaining disk (10) }\end{array}$ & $\begin{array}{l}\text { Central axis of the right } \\
\text { rotating disk (9) }\end{array}$ \\
\hline Mate 6 & Coincident & $\begin{array}{l}\text { Central point on axis } \mathrm{O}_{1-2} \text { of } \\
\text { the right retaining disk (10) }\end{array}$ & $\begin{array}{l}\text { Point on central axis of } \\
\text { the right rotating disk ( } 9 \text { ) }\end{array}$ \\
\hline
\end{tabular}




\begin{tabular}{|c|c|c|c|}
\hline Mate 7 & $\begin{array}{c}\text { Gear mate } \\
\text { ratio=1 }\end{array}$ & $\begin{array}{c}\text { Central axis of the left } \\
\text { rotating disk (9) }\end{array}$ & $\begin{array}{c}\text { Central axis of the right } \\
\text { rotating disk (9) }\end{array}$ \\
\hline $\begin{array}{c}\text { Mates } \\
8 \div 15\end{array}$ & Coincident & $\begin{array}{c}\text { Lefts points } 1 \div 8 \text { of the radial } \\
\text { slots semispheres } \\
16 \div 23\end{array}$ & $\begin{array}{c}\text { Central points of the left } \\
\text { balls } 1 \div 8\end{array}$ \\
\hline Mates 24 & Coincident & $\begin{array}{c}\text { Right points } 1 \div 8 \text { of the } \\
\text { radial slots semispheres }\end{array}$ & $\begin{array}{c}\text { Central points of the right } \\
\text { balls } 1 \div 8\end{array}$ \\
\hline Mate 25 & Coincident & $\begin{array}{c}\text { Front plane of the left rotating } \\
\text { disk (9) }\end{array}$ & $\begin{array}{c}\text { Front plane of the right } \\
\text { disk }(10) \text { and the retaining } \\
\text { generated as a single part }\end{array}$ \\
\hline Mate 26 & Parallel & $\begin{array}{c}\text { Front plane of the left rotating } \\
\text { disk (9) }\end{array}$ & $\begin{array}{c}\text { Right plane of the } \\
\text { assembly }\end{array}$ \\
\hline
\end{tabular}

The mates $8 \div 23$ were only necessary to specify the initial position of the balls. Mate 24 was used to specify the basic locations of the retaining disk (10), the box (11) and the horizontal table. Lastly, mates 25 and 26 were involved to specify the initial position and the direction of the first ball. Before starting the motion analysis calculation all these mates were suppressed.

The analysis time of the study was imposed to $0.3 \mathrm{~s}$. Within this time, the slotted disks are rotating 7.5 times, the duration of a complete rotation being $0.04 \mathrm{sec}$.

\section{Results and Discussion}

In the first stage, the coordinates, the velocities and the accelerations of a ball (no. 1 in Figure 1) where computed for a complete rotation of the slotted disk, the obtained outcomes being compared with the analytical calculations performed with Eq.'s (3)- (9). The obtained results for $R, x, v_{x}, v_{y}, a_{x}$ and $a_{y}$ as functions of the rotation angle $\alpha$ are shown in Figures 5, 6 and 7 .

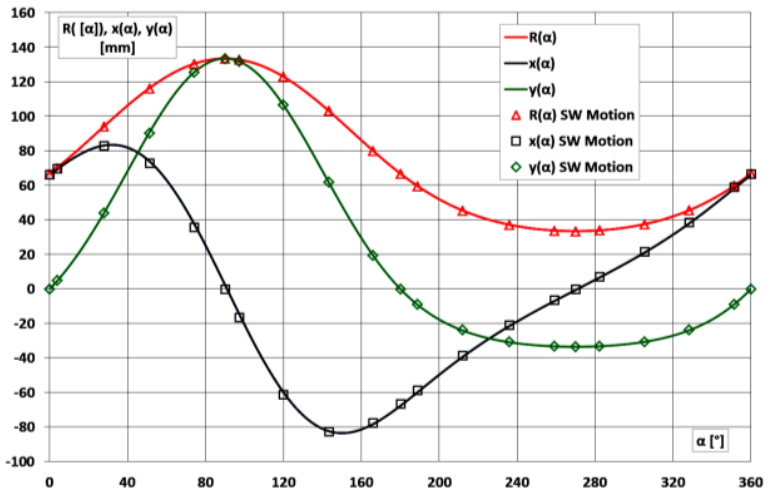

Figure 5

Comparison of the coordinates of a ball resulted from analytical calculation and SW simulation 


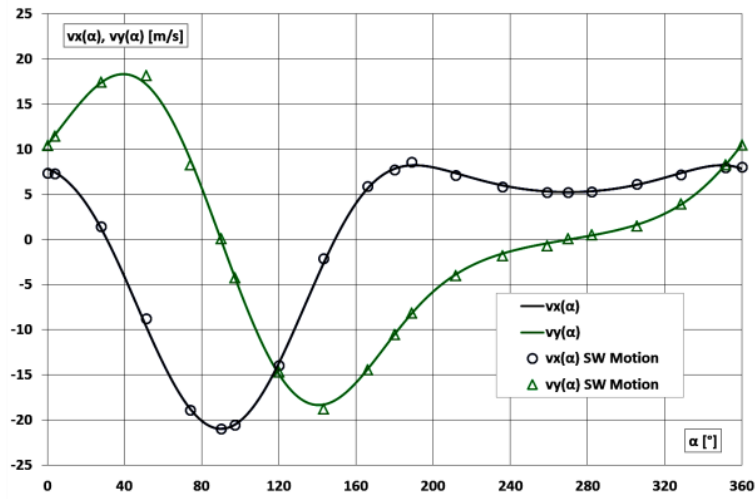

Figure 6

Comparison of the velocities of a ball resulted from analytical calculation and SW simulation

The simulation results were generated after the slotted disks (9) have performed a complete rotation $(\mathrm{T} \geq 0.04 \mathrm{~s})$ and when the steel ball remains in contact with the retaining disk (10).

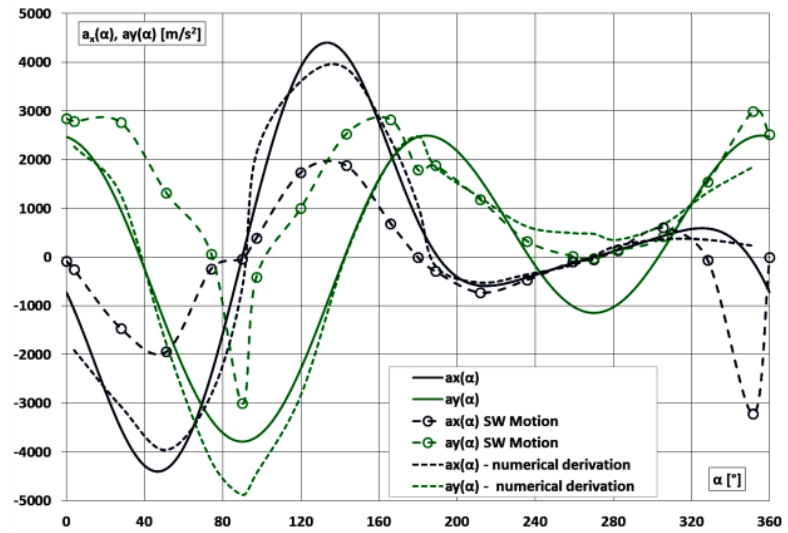

Figure 7

Comparison of the accelerations of a ball resulted from analytical calculation, SW simulation and numerical derivation of the velocities

One can see that the results obtained by analytical calculation and SW simulation are identical for the coordinates and the velocities of the balls, which can be assimilated as a calibration of the virtual model. In case of the accelerations, some differences between the analytical approach and SW simulation may be observed, discrepancies being higher for the accelerations in $\mathrm{X}$ direction, where the $\mathrm{x}$ component of the forces, acting on the balls of the two constructive groups, placed in mirror and rotating in opposite directions, are cancelling each the other. 
However, the shape of the variation curves is similar for the analytical and simulation approaches. Additionally, the graph includes the fluctuation of the accelerations obtained by numerical derivation of the velocities deducted by SW simulation, the deviations related to the results obtained by analytical calculation being minor.

These differences between the analytical and simulation approach may be explained by the fact that the simulation takes into account the effect of the collision of the balls during their contact with the inner bore of the retaining disk, a collision that generates an impact force which determines the acceleration values obtained by simulation, unlike the analytical calculation, where this impact effect was not taken into account.

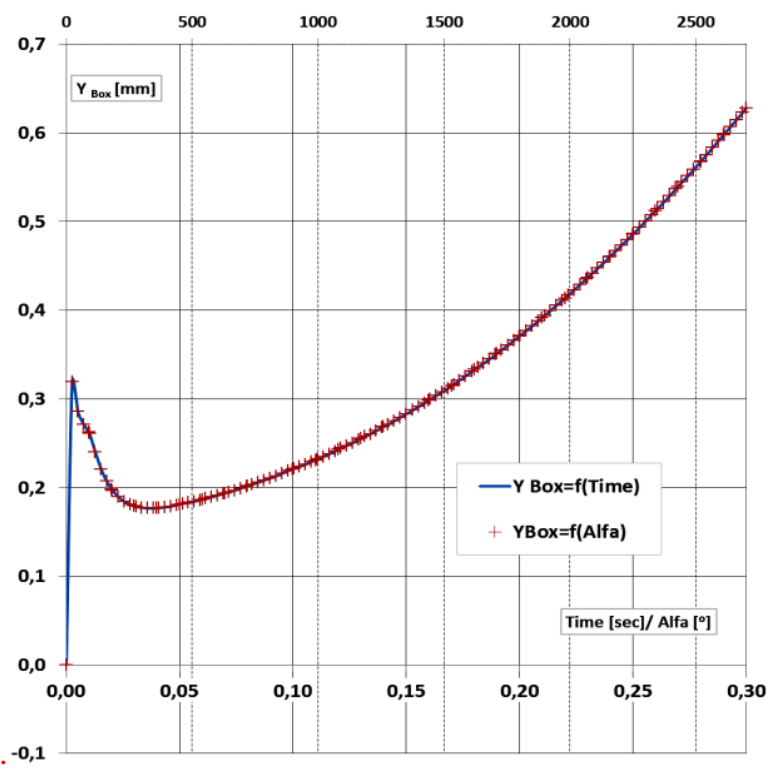

Figure 8

Simulation of the box displacement

The displacement and velocity of the box (11) were also simulated. The results for the displacement are presented in Figure 8, both as function of time and as function of the slotted disk rotation angle $\alpha$, while the velocity of the box in Y direction is described as a function of time in Figure $9 \mathrm{a}$.

Analyzing Figure 9b, one can observe that the box velocity graph $\left(v_{y} B o x\right)$ also includes negative values during the first rotation of the system $(T \leq 0.04 \mathrm{~s})$. The backward movement is due to the systems inertia and the impact between the balls and the retaining disc, which were considered in the simulation, phenomena's that were not included in the analytical relations. 

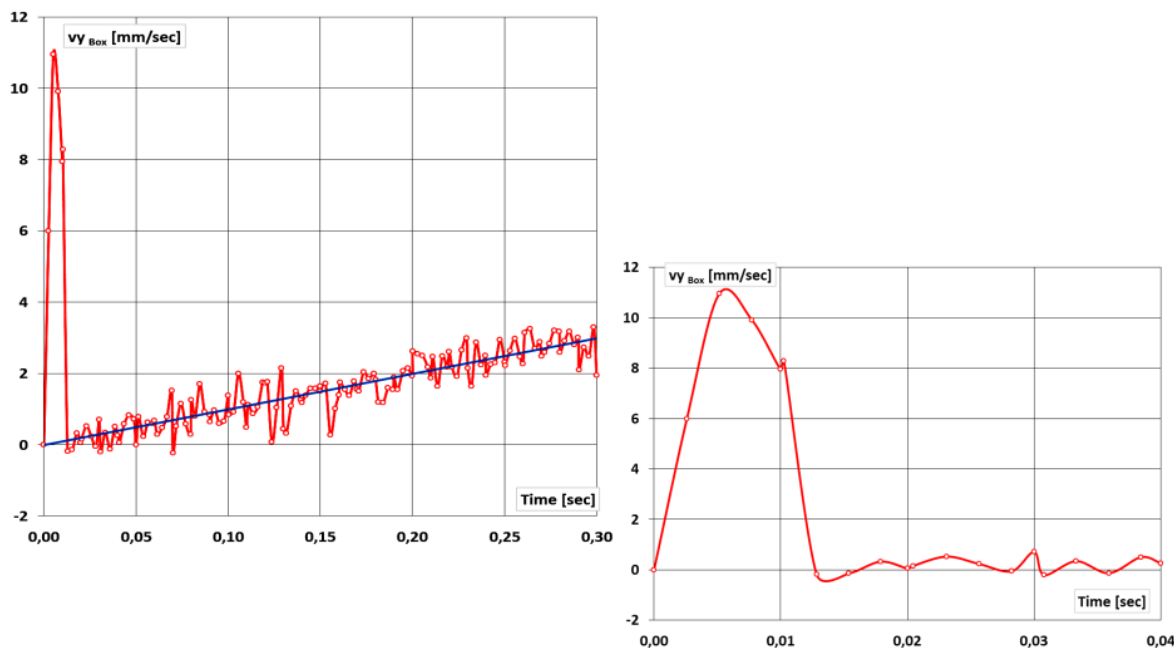

a)

b)

Figure 9

Simulation of the box velocity (a) entire simulation time; (b) first rotation of the system

In this motion simulation, the starting positions of the slotted disks were those where the balls no. 1 and 5 were oriented along the $X$ axis of the box. Furthermore, all the balls were initially placed at the radial slots beginning (close to the disk axis of symmetry). As it can be observed, immediately after initializing the rotation of the slotted disks, due to the high inertia of the system, the box increases its speed very quickly $\left(v_{y}=11 \mathrm{~mm} / \mathrm{s}\right)$ in a short time $(T<0.01 \mathrm{~s})$, after which, at about $0.04 \mathrm{~s}$ after starting, the system stabilizes, the speed of the box increasing linearly (trend line marked with blue in Figure 9a).

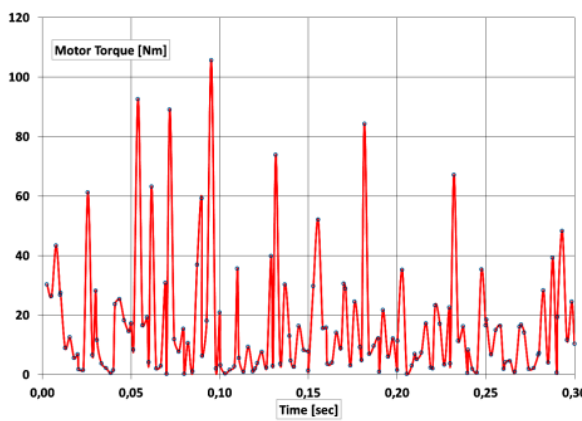

a)

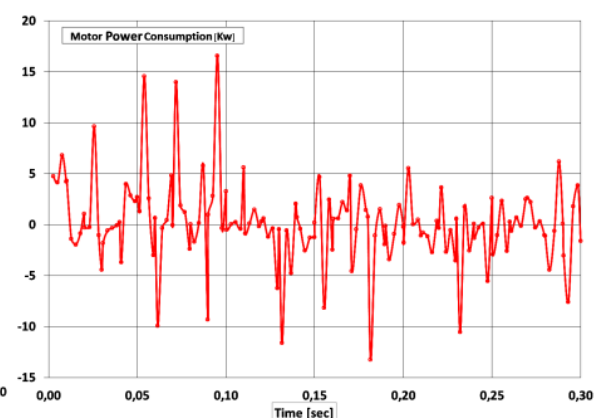

b)

Figure10

Simulation of motor torque (a) and power consumption (b) for driving the system 
Another major advantage of the Motion module from SolidWorks is that it provides in a fast way information regarding the torque variation of the motor and the power consumption, data that are harder to obtain by analytical approaches. Relevant information on these topics are presented in Figure 10. The obtained results confirm the previous finding that, during the first turns of the slotted disks, the propulsion system is unsteady. After the dynamic stabilization has occurred, the motor torque and the power consumption are decreasing significantly.

\section{Conclusions}

The paper presents the results of a kinematic and dynamic study of an IPS which uses the eccentric rotation of $8+8$ steel balls (active masses) to generate a one-way propulsion force.

The geometrical coordinates of one ball its velocities and accelerations were calculated by analytical equations and were graphically compared with the simulation results performed in SolidWorks Motion software. The results for both approaches have produced good agreement.

Furthermore, the simulation allowed us to observe that, immediately after starting, due to the inertia of the system and the impact between the balls and the retaining disk, the operation is unsteady with stabilization occurring after about $0.04 \mathrm{~s}$. Subsequently, the oscillations of the propulsion speed (velocity of the box in $\mathrm{Y}$ direction) decrease, together with the power consumption. During the $0.3 \mathrm{~s}$ of the simulation time the box (11) travelled a distance of $0.627 \mathrm{~mm}$, reaching an average velocity of $3 \mathrm{~mm} / \mathrm{s}$. This movement was also confirmed by the animation generated by SolidWorks Motion. Moreover, the present study proves that the IPS proposed by the authors is functional and capable to generate unidirectional linear movement. The results of the work herein, encourage future research and investigations for optimizing the system.

\section{References}

[1] N. L. Dean: System for converting rotary motion into unidirectional motion, US Patent 2886976, 1959

[2] I. A. Loukanov: Inertial propulsion of a mobile robot. IOSR Journal of "Mechanical and Civil Engineering", 2015, Vol. 12, No. 2, Version. 2, pp. 23-33

[3] C. G. Provatidis: A Device that can Produce Net Impulse Using Rotating Masses, Engineering, 2010, No. 2, pp. 648-657

[4] C. G. Provatidis: Repeated Vibrational Motion Using an Inertial Drive, Vibration and Acoustics Research Journal, 2019, Vol. 1, No. 1 pp. 27-43

[5] I. A. Lukanov, V. G. Vitliemov I. V. Ivanov: Dynamics of a Mobile Mechanical System with Vibration Propulsion (VibroBot), International Journal of Research in Engineering and Science, 2016, Vol. 4, No. 6, pp. 44-51 
[6] G. Anand, J. Jobin, K. Vijayan: Optimization of Configuration of Inertial Propulsion System for Future Space Application, American International Journal of Research in Science, Technology, Engineering \& Mathematics, 2014, Vol. 7, No. 2, pp 95-100

[7] E. Shimshi: Apparatus for energy transformation and conservation, US Patent 5673872, 1997

[8] A. W. Farrall: Inertial propulsion device, US Patent 3266233, 1966

[9] J. D. Mendez Llamozas Direct push propulsion unit, US Patent 2636340, 1953

[10] H. D. Kellogg: Gyroscopic inertial space drive, US Patent 3203644, 1965

[11] P. Haller: Propulsion Apparatus, US Patent 3177660, 1965

[12] J. D. Booden: Electromagnetically actuated thrust generator, US Patent 5782134,1998

[13] P. Haller: Propulsign Apparatus, US Patent 3177660, 1965

[14] N. J. Schnur: Method and apparatus for propelling an object by an unbalanced centrifugal force with continuous motion, US Patent 3979961, 1976

[15] A. Geröcs and. Z. I. Koka: Inertia drive system, Patent application no. RO133571-A2, 2019

[16] A. Geröcs, Z. I. Korka, G. R. Gillich: Analytical investigations on the influence of the geometry of an inertial drive on the propulsion force, Annals of "Eftimie Murgu" University of Reşiţa, Vol. 26, No. 1, 2019, pp. 76-85

[17] A. Geröcs, Z. I. Korka, I. Biró, V. Cojocaru: Analytical investigation of an inertial propulsion system using rotating masses, Journal of Physics: Conference Series, Volume 1426, 2020, 012031

[18] D. Nedelcu, G. R. Gillich, A. Bloju, I. Padurean: The Kinematic and Kinetostatic Study of the Shaker Mechanism with SolidWorks Motion, Journal of Physics: Conference Series, Volume 1426, 2020, 012025

[19] D. Nedelcu, M. D. Nedeloni, D Daia: The Kinematic and Dynamic Analysis of the Crank Mechanism with SolidWorks Motion, Proceedings of the $11^{\text {th }}$ WSEAS International Conference on Signal Processing, Computational Geometry and Artificial Vision, Florence, Italy, August 23-25, 2011, pp. 245-250

[20] Dassault Systems 2010 SolidWorks 2010 Motion, 300 Baker Avenue, Concord, Massachusetts, 